\title{
Relative 3D Regularized B-spline Surface Reconstruction Through Image Sequences
}

\author{
ChangSheng Zhao and Roger Mohr \\ LIFIA-INRIA \\ 46 Avenue Félix Viallet 38031 Grenoble cedex, France
}

\begin{abstract}
This paper considers the problem of 3D surface reconstruction using image sequences. We propose a direct method of surface reconstruction which is based on regularized uniform bicubic B-spline surface patches. The reconstruction is achieved by observing the motion of occluding contours $[1,4,10]$, i.e., where the view lines graze the surface. It has been shown that reconstruction of such a $3 \mathrm{D}$ surface is possible when the camera motion is known, with the exception of those fully concave parts where no view line can be tangential to the surface. This approach differs from previous work, it states directly the problem of regularization on the $3 \mathrm{D}$ surface. Experimental results are presented for real data.
\end{abstract}

\section{Introduction}

The problem of representing and reconstructing 3D surfaces has received an enormous amount of attention in computer vision research over the past decade. The interest arises at least in part because robust surface reconstruction would have applicability to a wide variety of fields namely, manufacturing automation, terrain mapping, vehicule guidance, surveillance, virtual reality, etc. But aside from any practical applications, the problem has a great deal of scientific and mathematical interest.

From the perspective of classical differential geometry, there are three representations for a surface of an object: explicit, implicit, and parametric [3]. In this paper, we use uniform bicubic B-spline surface patches to give a parametric representation of a surface. Our aim is to reconstruct the $3 \mathrm{D}$ surface of an object from the observation of occluding contours $[1,4,6,7,10]$ together with the knowledge of the motion of the camera mounted on the robot. We assume that the camera is modeled as a pinhole. We suppose that the object is smooth, specifically, that its surface is at least $C^{2}$. Our original contributions with respect to these related works are twofold: first, we introduce a direct regularization of the $3 \mathrm{D}$ surface to be reconstructed instead of smoothing the contours in the $2 \mathrm{D}$ image. This is based on the regularized uniform bicubic B-spline surface patches. Secondly, we propose global surface recovery from small local patches. Previous authors were satisfied with the recovery of only some local properties of the surface such as curvature or normal curvature estimation.

The paper is composed of three parts: in the first part we will introduce the main basic notions used here; i.e., the uniform bicubic B-spline surface patches, 
the definitions of the occluding contour and the epipolar parameterisation of a surface. The second part describes mathematically our method for B-spline surface patches reconstruction. In the last part, we present experimental results on real data and discuss their accuracy.

\section{Definitions and notations}

\subsection{Uniform bicubic B-spline surface patches}

B-spline curves are piecewise polynomial functions. These curves are defined by a sequence of control points, and pass near to those points. For more detail see [2]. In practical applications, cubic B-spline curves are the most frequently used, and play a important role. In this section, we will only concentrate on the uniform bicubic B-spline surface patches, a natural and straightforward generalization of the uniform cubic B-spline curves. The uniform bicubic B-spline surface patches can be expressed by the following double summation:

$$
X(s, t)=\sum_{i=-3}^{m-1} \sum_{j=-3}^{n-1}\left(\alpha_{i j} B_{i}(s) B_{j}(t), \beta_{i j} B_{i}(s) B_{j}(t), \gamma_{i j} B_{i}(s) B_{j}(t)\right)
$$

where $s, t \in I \times J \subseteq I R \times I R,\left(\alpha_{i j}, \beta_{i j}, \gamma_{i j}\right)$ are called control points, the product $B_{i}(s) B_{j}(t)$ is called tensor-product $\mathrm{B}$-spline. $B_{i}, B_{j}$ are simply the basis functions of uniform cubic B-spline curves [8].

They have two following interesting properties: tensor-product B-splines have local compact support. B-spline surface patches transform as their control points under affine transforms.

\subsection{Occluding contour}

Now we consider an object surface $S$. The camera model is assumed to be the pinhole, its center of projection is at $O$. The occluding contour $w$ of a surface $S$, viewed from a point $O$, is defined to be the projection of the set of points $W$ on the surface $S$ for which $\boldsymbol{n} . \boldsymbol{O W}=0$, where $\boldsymbol{n}$ is the unit normal vector corresponding to the tangent plane at $W$.

\subsection{Epipolar parameterisation}

The parameterisation of the spatio-temporal surface and the parameterisation of the object surface are directly linked. We choose two parameters $t$ and $s: t$ is a temporal parameter, i.e., the parameter $t$ is linked to the motion of the camera. The parameter $s$ is spatial, it is imposed by the arc length of the occluding contour in the image, the value of $s$ is linked from one image to the other through the epipolar constraint (see Figure 1). So locally in a sequence each point on a. surface is unambiguously parametrised, except for the points where the epipolar lines are tangential to the object surface. The choice we made in our work is the epipolar parameterisation described by Cipolla \& Blake [4]. 


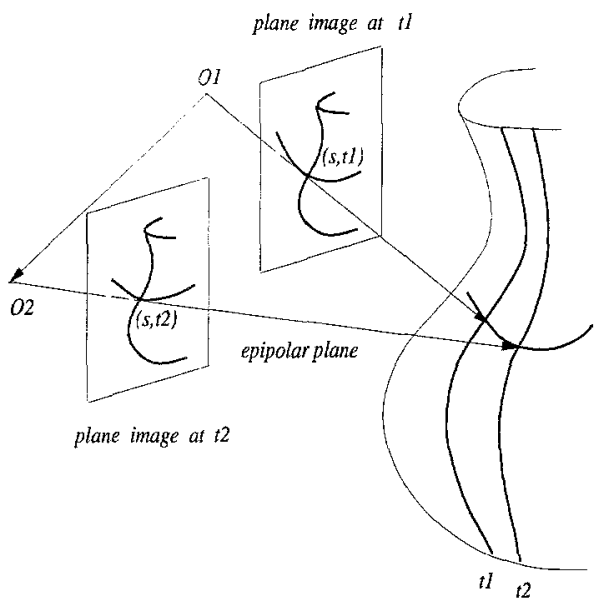

Fig. 1. Epipolar parameterisation

\section{Mathematical model}

We assume that the parametric representation of a surface is defined by

$$
\begin{aligned}
\boldsymbol{X}(s, t) & =\left(X_{1}(s, t), X_{2}(s, t), X_{3}(s, t)\right) \\
& =\sum_{i=-3}^{m-1} \sum_{j=-3}^{n-1}\left(\alpha_{i j} B_{i}(s) B_{j}(t), \beta_{i j} B_{i}(s) B_{j}(t), \gamma_{i j} B_{i}(s) B_{j}(t)\right) .
\end{aligned}
$$

Uniform bicubic B-spline surface patches reconstruction requires then the estimation of $3 \times(m+3) \times(n+3)$ unknowns $\alpha_{i j}, \beta_{i j}, \gamma_{i j}, i=-3, \ldots, m-1$, $j=-3, \ldots, n-1$ in the formula (2).

\subsection{Two linear equations}

We suppose that a point $w$ with the coordinates $(x, y)$ is the projection of a point $W$ with the homogeneous coordinates $\left[X_{1}(s, t), X_{2}(s, t), X_{3}(s, t), 1\right]$ on the surface $S$, under the projection matrix $M=\left(m_{p r}\right)_{p=1, \ldots, 3, r=1, \ldots, 4}$.

We have for homogeneous coordinates:

$$
\left\{\begin{array}{l}
x=\frac{m_{11} X_{1}(s, t)+m_{12} X_{2}(s, t)+m_{13} X_{3}(s, t)+m_{14}}{m_{31} X_{1}(s, t)+m_{32} X_{2}(s, t)+m_{33} X_{3}(s, t)+m_{34}} \\
y=\frac{m_{21} X_{1}(s, t)+m_{22} X_{2}(s, t)+m_{23} X_{3}(s, t)+m_{24}}{m_{31} X_{1}(s, t)+m_{32} X_{2}(s, t)+m_{33} X_{3}(s, t)+m_{34}} .
\end{array}\right.
$$

Trivially, (3) can be rewritten after substituting (2) and multiplying by the appropriate denominator as

$$
\left\{\begin{array}{l}
P_{1}\left(x, s, t, \alpha_{i j}, \beta_{i j}, \gamma_{i j}, m_{p r}\right)=0 \\
P_{2}\left(y, s, t, \alpha_{i j}, \beta_{i j}, \gamma_{i j}, m_{p r}\right)=0
\end{array}\right.
$$


It is very easy to check that the two equations (4) are linear in the unknowns $\alpha_{i j}, \beta_{i j}, \gamma_{i j}$.

We can compute the view line corresponding to a given point in the image when we know its coordinates and the projection matrix from two equations (3).

\subsection{One additional nonlinear equation}

From now on, we can write a nonlinear equations system of the surface reconstruction in the following way.

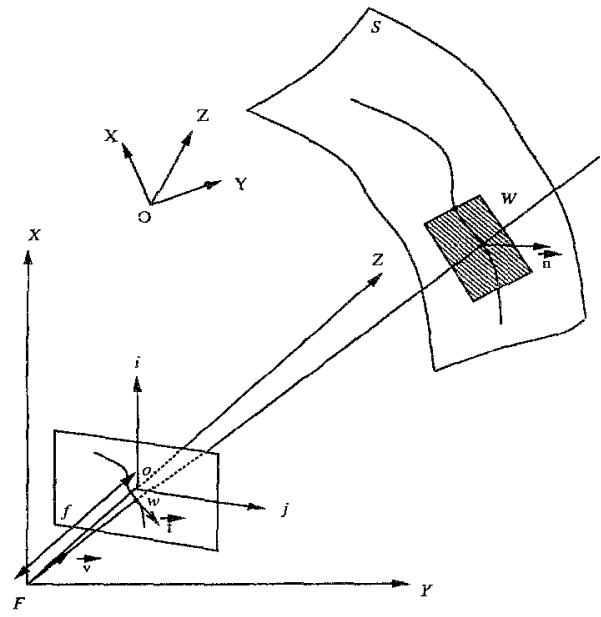

Fig. 2. Property of an occluding contour

Let $W$ be a point on the surface $S$ (see Figure 2). A point $w=(x, y)$ of the occluding contour in the image plane is the projection of the point $W=$ $\left(X_{1}(s, t), X_{2}(s, t), X_{3}(s, t)\right)$. The optical ray $v=\left(v_{1}, v_{2}, v_{3}\right)$ determined by $F W$ is then tangent to the object surface $S$. The normal vector $\boldsymbol{n}$ to the tangent plane at $W$ is given by

$$
\frac{\partial \boldsymbol{X}(s, t)}{\partial s} \times \frac{\partial \boldsymbol{X}(s, t)}{\partial t}
$$

where " $x$ " denotes the cross product.

According to the definition of an occluding contour, we have the following relation:

$$
\boldsymbol{n} \cdot \boldsymbol{v}=\left(\frac{\partial \boldsymbol{X}(s, t)}{\partial s} \times \frac{\partial \boldsymbol{X}(s, t)}{\partial t}\right) \cdot \boldsymbol{v}=\left|\begin{array}{lll}
\frac{\partial X_{1}}{\partial s_{1}} & \frac{\partial X_{2}}{\partial s} & \frac{\partial X_{3}}{\partial s} \\
\frac{\partial X_{1}}{\partial t} & \frac{\partial X_{2}}{\partial t} & \frac{\partial X_{3}}{\partial t} \\
v_{1} & v_{2} & v_{3}
\end{array}\right|=0 .
$$

Equation (6) can be rewritten as follows by substituting (2) and (5)

$$
P_{3}\left(x, y, s, t, \alpha_{i j}, \beta_{i j}, \gamma_{i j}, m_{p r}\right)=0 .
$$


Unfortunately, this equation (7) is not linear in the unknowns $\alpha_{i j}, \beta_{i j}, \gamma_{i j}$.

As the tangent plane to the surface at $W$ is defined by the optical ray $v$ and the tangent $\boldsymbol{t}$ to the occluding contour at $w$, we have then the following relation:

$$
(\boldsymbol{v} \times \boldsymbol{t}) \times \boldsymbol{n}=0 .
$$

But this equation (8) gives the same information as equation (6). Indeed, it describes also the orthogonal property between the normal vector and the tangent plane to the surface at $W$. Therefore, it is omitted.

Finally, we can regroup these equations (4) and (7) into the following system of three equations:

$$
\left\{\begin{array}{l}
P_{1}\left(x, s, t, \alpha_{i j}, \beta_{i j}, \gamma_{i j}, m_{p r}\right)=0 \\
P_{2}\left(y, s, t, \alpha_{i j}, \beta_{i j}, \gamma_{i j}, m_{p r}\right)=0 \\
P_{3}\left(x, y, s, t, \alpha_{i j}, \beta_{i j}, \gamma_{i j}, m_{p r}\right)=0 .
\end{array}\right.
$$

From the above system, we notice that $s, t$ are two parameters of B-spline surface patches, $(x, y)$ are coordinates of a point of an occluding contour in an image. According to the epipolar parameterisation of a spatio-temporal surface, $s$ is only the arc length of the occluding contour of the point $(x, y)$ at $t$ time in an image sequence when its origin is chose by first point extracted of the occluding contour.

\section{Solving the system}

We can generalize the above system for a sequence of images. Let $k$ be the number of image in the sequence. Let $l$ be the number of point extracted from the occluding contour of the $k^{\text {th }}$ image of the sequence. We have then the following system of equations:

$$
\left\{\begin{array}{l}
q_{1}\left(x_{l}^{k}, s_{l}^{k}, t_{l}^{k}, \alpha_{i j}, \beta_{i j}, \gamma_{i j}, m_{p r}^{k}\right)=0 \\
q_{2}\left(y_{l}^{k}, s_{l}^{k}, t_{t}^{k}, \alpha_{i j}, \beta_{i j}, \gamma_{i j}, m_{p r}^{k}\right)=0 \\
q_{3}\left(x_{l}^{k}, y_{l}^{k}, s_{l}^{k}, t_{l}^{k}, \alpha_{i j}, \beta_{i j}, \gamma_{i j}, m_{p r}^{k}\right)=0 .
\end{array}\right.
$$

As there are $k_{o}$ images and $l_{o}$ points on each image, this leads us to $3 \times k_{o} \times l_{o}$ equations. The unkowns are $3 \times(m+3) \times(n+3)$. So if $k_{o}$ and $l_{o}$ are large enough, the system of equations (10) is redundant.

The most direct procedure is to try to solve this system of nonlinear equations. We can hope to solve it by standard least squares technique. The problem can be formulated as minimization over

$$
E=\sum_{k=1}^{k_{o}} \sum_{l=1}^{l_{0}} \lambda\left(q_{1}^{2}+q_{2}^{2}\right)+\mu q_{3}^{2}
$$

where $\lambda, \mu \in I R^{+}$are two weight parameters. 
Since there is some noise, introduced by errors or approximations at the contour detection stage, the above method does not allow the shape of the surface to be easily adjusted. We therefore introduce a regularization term on the surface $[8,11]$. The term allows us to minimize the total variation in the local surface orientation. This suggests that the term should measure some factor of the second-order derivatives of the surface.

Finally, the regularized B-spline surface patches functional can be written as

$$
E=\sum_{k=1}^{k_{0}} \sum_{l=1}^{l_{0}}\left[\lambda\left(q_{1}^{2}+q_{2}^{2}\right)+\mu q_{3}^{2}\right]+\tau \int_{I} \int_{J}\left[\left(\frac{\partial^{4} X_{1}}{\partial^{2} s \partial^{2} t}\right)^{2}+\left(\frac{\partial^{4} X_{2}}{\partial^{2} s \partial^{2} t}\right)^{2}+\left(\frac{\partial^{4} X_{3}}{\partial^{2} s \partial^{2} t}\right)^{2}\right] d s d t
$$

where $\tau \in I R^{+}$is called the tensor of B-spline surface patches. Indeed, the regularization term goes back to minimize the variation of the curvature. This allows to smooth the surface of the regions where there is noise.

Currently, two standard numerical least squares methods, quasi-Newton and Levenberg-Marquardt are implemented in our system. B-spline surface patches are completely determined by a set of control points, and we know that these control points are close to the object surface in space. Therefore we can compute the depth of some points on the surface by a standard triangulation method. The starting values of the system are then provided by the depth points. Experiments show that the Levenberg-Marquardt's algorithm converges better than the quasiNewton's algorithm.

\section{$5 \quad$ Experimental results}

In our experiments we made use of a camera, with a $18 \mathrm{~mm}$ lens. The camera is assumed to be a perfect pinhole. In practice, the accuracy of the projection matrix (translation and rotation) does not reach the required precision. We therefore want to use stable points in the scene for computing a reliable projection matrix. We have built a transparent cube and placed a carafe inside (see Figure 3 ). The polyhedral pieces of paper (white and black) have been added to the scene in order to get accurate epipolar geometry [9]. The contour points were obtained by a standard gradient based edge detector [5], and these contours were approximated with cubic B-splines [8]. The corners of the polyhedral pieces of paper in the scene were detected by a method developed in our laboratory. Then these corners were tracked by the standard correlation operator. The centers of the eight balls were computed as the intersection points of the medians of the bars. Finally, we chose an absolute coordinate frame attached to the cube. The accurate projection matrices of the sequence were thus computed.

Figure 3 shows the first and the last images of the sequence of ten images of a carafe.

Figure 4 shows results of the reconstructed B-spline surface patches of the carafe using a sequence of ten images. The parameters in this process are: $m=3$, $n=1, k=10, l=70$. So the number of equations in our redundant system is 

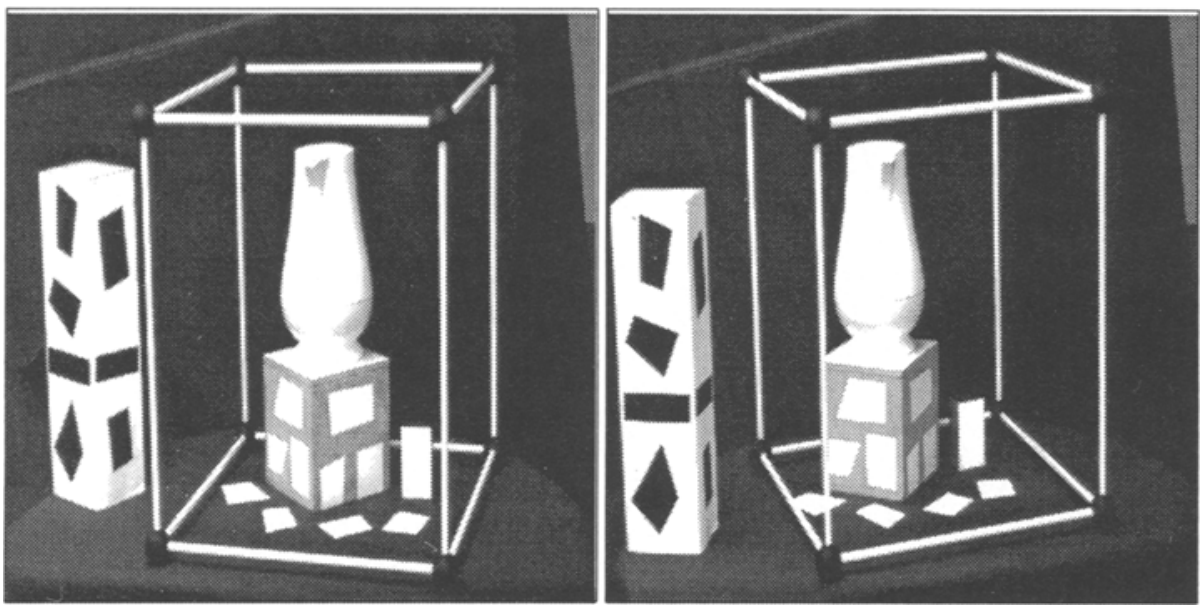

Fig. 3. The first (left) and the last (right) images of the sequence

$3 \times k \times l=2100$, the number of unknowns is $3 \times(m+3) \times(n+3)=72$, and the number of the control points of the reconstructed B-spline surface patches is 24 . The necessary number of iterations of Levenberg-Marquardt's algorithm is only 3. In particular, the total time for 3 iterations is 99 seconds on a SunSparc10. We remark in Figure 4 that the quality of the reconstruction surface is qualitatively good. Our final goal is to reconstruct the whole surface when the camera makes a tour of the carafe.
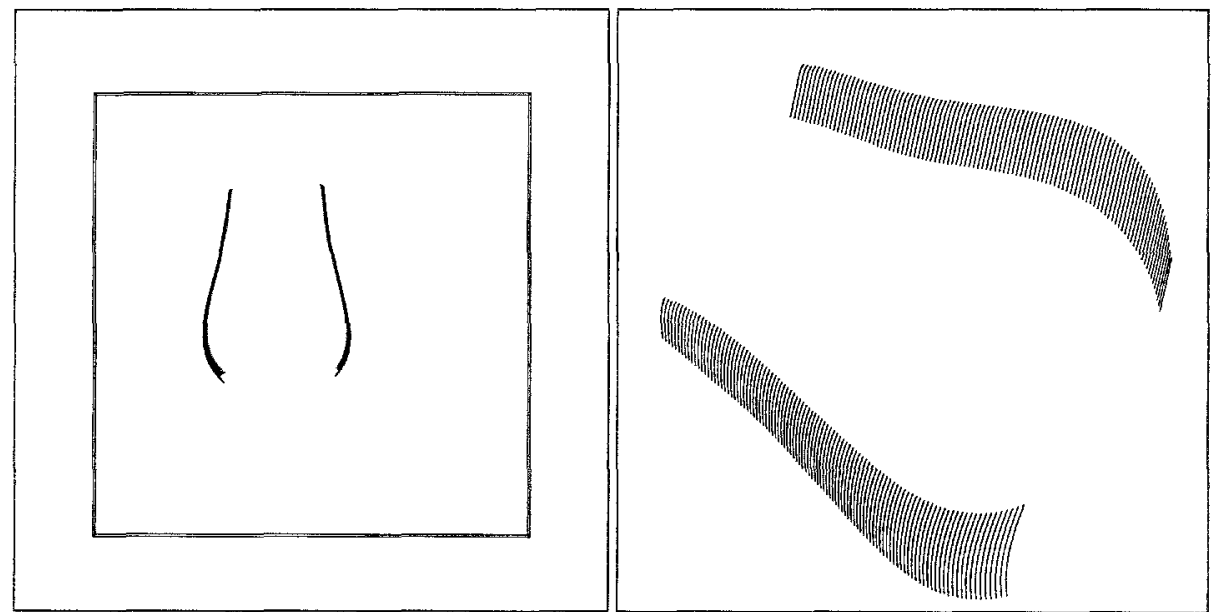

Fig. 4. Two reconstructed B-spline surface patches of the carafe are displayed with the transparent cube (left); A view of two reconstructed B-spline surface patches are displayed without the transparent cube (right) 
Figure 5 shows the graphs of Gaussian and mean curvatures for the estimated $\mathrm{B}$-spline surface patches of our carafe. We notice in Figure 5 that the values of the Gaussian curvature of some points on the B-spline surface patches are negative.
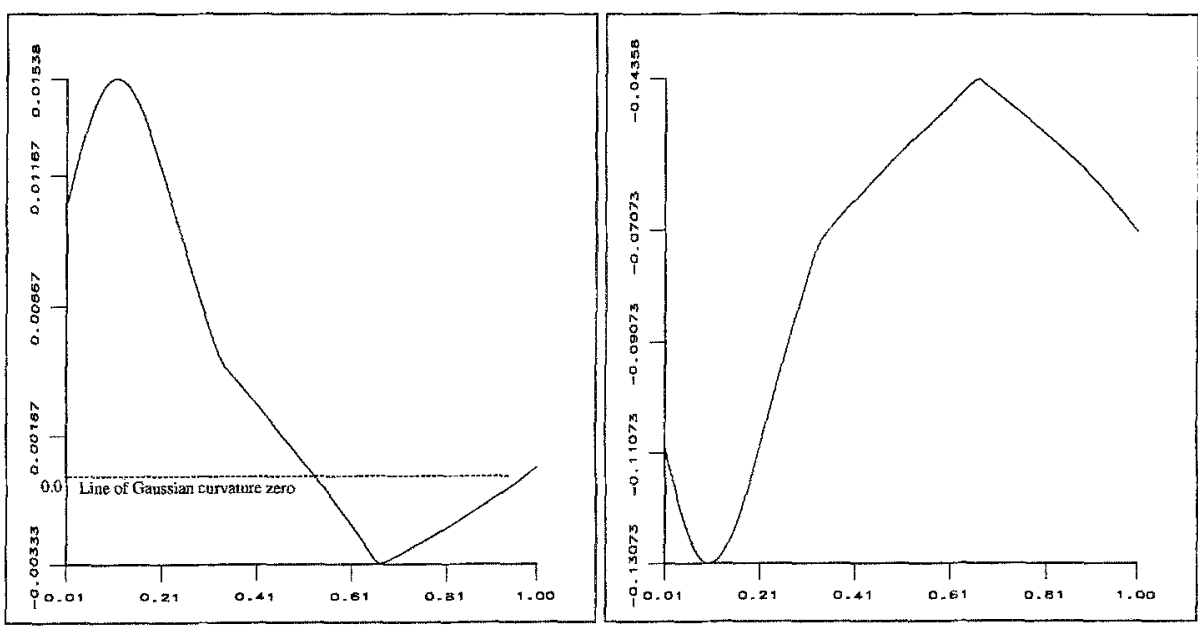

Fig. 5. Graphs of Gaussian (left) and mean (right) curvatures of the reconstructed B-spline surface patches of the carafe

In order to verify the quality of our reconstructed carafe, we compare the real diameter and the measured diameter. Measures of diameter of our carafe are performed with a pair of sliding calipers.

Table 1 gives the diameter of the carafe and the corresponding computed diameter. The maximal error is $5.40 \mathrm{~mm}$. The mean error is $3.83 \mathrm{~mm}$.

\begin{tabular}{|c|c|c|c|}
\hline Height & Real diameter & Computed diameter & Error \\
\hline 60.0 & 144.0 & 139.2 & 4.8 \\
70.0 & 148.5 & 143.1 & 5.4 \\
80.0 & 150.2 & 147.5 & 2.7 \\
90.0 & 148.5 & 149.2 & -0.7 \\
100.0 & 146.0 & 148.8 & -2.8 \\
110.0 & 142.0 & 146.6 & -4.6 \\
120.0 & 139.5 & 143.9 & -4.4 \\
130.0 & 135.0 & 140.3 & -5.3 \\
\hline
\end{tabular}

Table 1. Errors of the diameter of our reconstructed carafe (units are $\mathrm{mm}$ )

Figure 6 shows the first and the last images of the sequence of ten images of a tube. Figure 7 shows results of the reconstructed B-spline surface patches of the tube using this images sequence. As the epipolar lines are almost tangential 

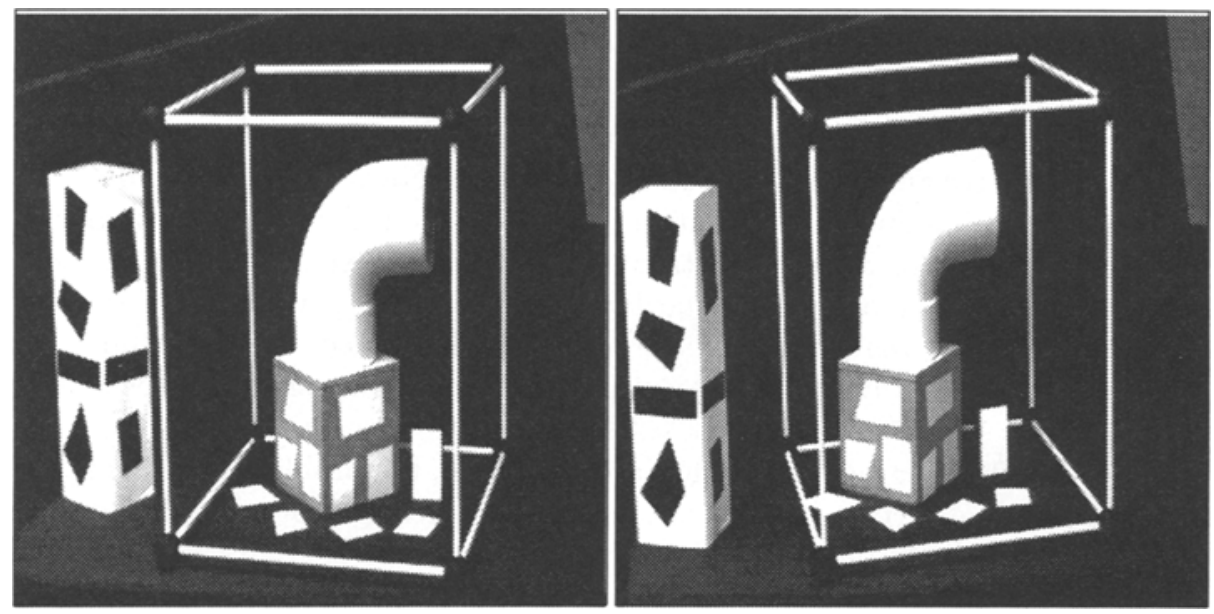

Fig. 6. The first (left) and the last (right) images of the sequence

to the top surface of the tube, this leads to the poor reconstruction displayed in Figure 7 . This is another example of the aperture problem.
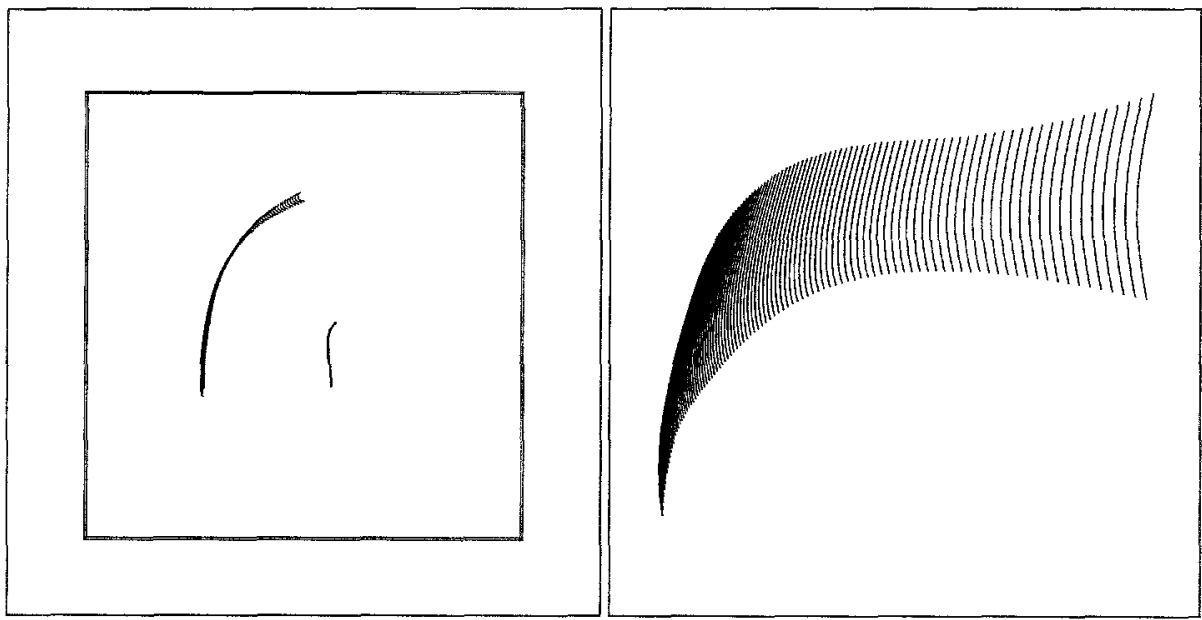

Fig. 7. Reconstructed B-spline surface patches of the tube is displayed with the transparent cube (left); A view of the reconstructed B-spline surface patches is displayed without the transparent cube (right) 


\section{Conclusion}

In this paper we have shown that the reconstruction of a 3D surface is possible when the camera motion is known. The reconstruction process provided good quantitative and qualitative results. We have shown also how the different parameters of B-spline surface patches reconstruction should be conveniently selected according to available data obtained through experiments. The iterative algorithm of Levenberg-Marquard works well, convergence can generally be obtained in two to ten iterations. We have actually tested our algorithm on a large number of real images representing several different shapes of occluding contours.

Once the B-spline surface patches are estimated, the problem of merging the different B-spline surface patches obtained with different motions still remains. This step is presently being completed.

Acknowledgements: We would like to thank Françoise Veillon, Long Quan, David Sinclair, Marie-Odile Berger, Radu Horaud, Boubakeur Boufama and Pascal Brand for their helpful conversations and suggestions. This work was supported by Esprit BRA project Second and by the French national project GDR-PRC.

\section{References}

1. Arbogast, E., Mohr R.: 3D structures inference from images sequences. International Journal of Pattern Recognition and Artificial Intelligence. 5(5) (1991) 749

2. Bartels, R.H., Beatty J.C., Barsky B.A.: An introduction to splines for use in computer graphics and geometric modeling. Morgan Kaufman Pu. Inc., (1987)

3. Do Carmo, M.P.: Differential geometry of curves and surfaces. Prentice Hall, (1976)

4. Cipolla, R., Blake, A.: Surface shape from the deformation of apparent contours. International Journal of Computer Vision. 9(2) (1992) 83-112

5. Deriche, R.: Using Canny's criteria to derive a recursively implemented optimal edge detector. International Journal of Computer Vision. 1(2) (1987) 167-187

6. Giblin, P., Weiss, R.: Reconstruction of surfaces from profiles. In Proceedings of the 1st International Conference on Computer Vision. London, England, (1987) $136-144$

7. Koenderink, J.J.: What does the occluding contour tell us about solid shape? Perception, 13 (1984) 321-330

8. Laurent, P.J.: Courbes ouvertes ou fermées par B-splines régularisées. Technical Report RR 652-M-, IMAG, Grenoble, France, (1987)

9. Mohr, R., Quan, L., Veillon, F., Boufama, B.: Relative 3D reconstruction using multiples uncalibrated images. Technical Report RT 84-I-IMAG LIFIA 12, LIFIAIMAG, (1992)

10. Vaillant, R., Faugeras, O.D.: Using Extremal Boundaries for 3-D Objet Modeling. IEEE Transactions on PAMI, 14(2) (1992) 157-173

11. Zhao, C.S., Mohr, R.: B-spline patches for surface reconstruction in computer vision. In P. J. Laurent, A. Le Méhanté, and L. L. Schumaker, editors, Curves and Surfaces II, Academic Press, Boston, 1994. 